\title{
Anaesthesia of the posterior urethra and pain reduction during cystoscopy - a randomized controlled trial
}

\author{
Sławomir Poletajew, Sylwia Bender, Paweł Pudełko, Marcin Łykowski, Tomasz Piecha, Bartosz Sutkowski, \\ Piotr Radziszewski \\ Department of Urology, Medical University of Warsaw, Warsaw, Poland
}

Videosurgery Miniinv 2017; 12 (1): 75-80

DOI: https://doi.org/10.5114/wiitm.2017.66506

\begin{abstract}
Introduction: Standard intra-urethral instillation of anaesthetic gel may not sufficiently exclude pain perception during cystoscopy.

Aim: To evaluate the impact of the anaesthesia within the posterior urethra on pain intensity related to cystoscopy in men. Material and methods: One hundred and twenty-seven men undergoing cystoscopy were prospectively enrolled in the study. Patients were randomly assigned to the experimental or control group (66 vs. 61 patients). Intra-urethral instillation of $2 \%$ lidocaine gel was done in both groups. In the experimental group, the posterior urethra was additionally anaesthetized with distribution of the lidocaine gel by catheterisation. The study endpoints were pain intensity at successive time points of the procedure assessed on a numeric rating scale, overall pain intensity assessed on a Likert scale, the need for analgesics during $6 \mathrm{~h}$ after the procedure, and the frequency of urinary tract infections (UTIS) during 14 days after the procedure.

Results: Pain perception during cystoscopy did not differ significantly between the two groups ( $p>0.05)$. However, after $6 \mathrm{~h}$ patients in the experimental group were more likely to declare that the cystoscopy was painless (81.8\% vs. $70.2 \%$, relative risk $=1.17)$. The need for analgesics and the incidence of UTI were similar in both groups $(p>0.05)$. Statistically significant differences regarding pain perception were observed depending on patients' age and the number of transurethral procedures performed in the past, with no relation to type of anaesthesia $(p<0.05)$.

Conclusions: Anaesthesia of the posterior urethra is not more efficacious in reducing pain related to cystoscopy than standard instillation of anaesthetic gel. However, it improves the general perception of the procedure, and hence may positively influence patients' compliance.
\end{abstract}

Key words: bladder cancer, cystoscopy, anaesthesia, urethra.

\section{Introduction}

Bladder cancer is the most common urinary tract malignancy [1]. About $75 \%$ of patients undergo transurethral resection of the bladder tumour (TURBT) due to the presence of non-muscle-invasive bladder cancer (NMIBC). Afterwards they all need close follow-up based on cystoscopy repeated every 3-12 months for at least 5 years [2].
Cystoscopy remains an uncomfortable procedure with inevitable pain reported by patients. The pain is usually classified as mild or moderate, may be reduced by the use of a flexible instrument, but still remains consistently reported by all patients [3, 4]. Moreover, while patients report less pain related to cystoscopy than they anticipate before the procedure, conversely physicians are likely to underestimate actual pain perceived by patients $[5,6]$. The 
pain is more intense in men than in women [4]. The most painful part of the procedure is insertion of a cystoscope, especially at the moment when it passes the external urethral sphincter $[7,8]$.

While the body of literature on the optimisation of urethral anaesthesia is growing, it is still questionable whether standard installation of anaesthetic gel or plain lubricant through the external urethral orifice can sufficiently exclude pain perception within the posterior urethra in men. Simultaneously, none of already published studies was aimed at design and evaluation of anaesthesia in this region before diagnostic cystoscopy.

\section{Aim}

The aim of the study was to evaluate the effect of the anaesthesia within the posterior urethra on the pain intensity during and after rigid cystoscopy in men.

\section{Material and methods}

One hundred and twenty-seven men with a mean age of 71.4 years and a history of TURBT, undergoing routine follow-up cystoscopy, were prospectively enrolled in the study. Patients were randomly assigned to the experimental or control group (66 vs. 61 patients) based on a randomization generator available at randomization.com. The institutional review board approved the study protocol. All patients gave written consent to participate in the study. Detailed characteristics of the study population are presented in Table I. Figure 1 presents the CONSORT diagram.

Twelve $\mathrm{ml}$ of $2 \%$ lidocaine gel, administered through the external urethral orifice, was used for local anaesthesia in both groups. In the experimental group, after the initial analgesic effect was achieved, additionally a $10 \mathrm{Fr}$ Tieman bladder catheter (coude- tipped catheter) was inserted to distribute the lidocaine gel along the entire length of the urethra, and hence to anaesthetize the posterior urethra. The time from gel administration to urethra instrumentation with the cystoscope was 5 min in both groups.

Urinary tract infection (UTI) was excluded before the procedure in all cases. Six residents in urology participated in the study and performed uniform anaesthesia and cystoscopies. No dedicated patient preparation for the procedure was adopted. All the cystoscopies were performed with a 17 Fr rigid cystoscope in a lithotomy position.

The primary study endpoint was the pain intensity at different time points during and after the procedure assessed on a 10-point numeric rating scale (NRS). Secondary study endpoints were overall intensity of pain related to the procedure assessed on a Likert scale, the need for analgesics within $6 \mathrm{~h}$ after the procedure, and the frequency of UTI within 14 days after the procedure.

We hypothesized that anaesthesia of the posterior urethra would be superior to standard local anaesthesia with respect to pain intensity at the time of insertion of the cystoscope and non-inferior with respect to other study end-points. We also assumed that the NRS score at the time of insertion of the cystoscope would be at least 30\% lower in the experimental group. Considering a mean visual analogue scale (VAS) score of 3.0 in the control group based on literature data, a sample size of 50 patients in each study arm was planned to detect a $30 \%$ difference in NRS score and obtain $80 \%$ power using a one-sided $\alpha$ value of 0.05 .

\section{Statistical analysis}

Statistical calculations were performed with Statistica 10.0 software. The Shapiro-Wilk test con-

Table I. Characteristics of the studied groups

\begin{tabular}{|lcc|}
\hline Parameter & Experimental group & Control group \\
\hline Number of patients & 66 & 61 \\
\hline Mean age [years] & 73.0 & 71.2 \\
\hline Mean time interval from the last TURBT [days] & 497 & 494 \\
\hline $\begin{array}{l}\text { Mean number of TURBT performed per patient } \\
\text { Number of patients who received adjuvant } \\
\text { intravesical BCG therapy after TURBT }\end{array}$ & 4.6 & 4.5 \\
\hline $\begin{array}{l}\text { Mean time interval from last intravesical } \\
\text { instillation of BCG [days] }\end{array}$ & 11 & 9.05 \\
\hline
\end{tabular}


Assessed for eligibility $(n=152)$

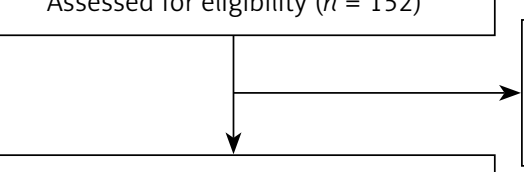

Excluded $(n=25)$

- Not meeting inclusion criteria $(n=17)$

- Declined to participate $(n=8)$

- Other reasons $(n=0)$

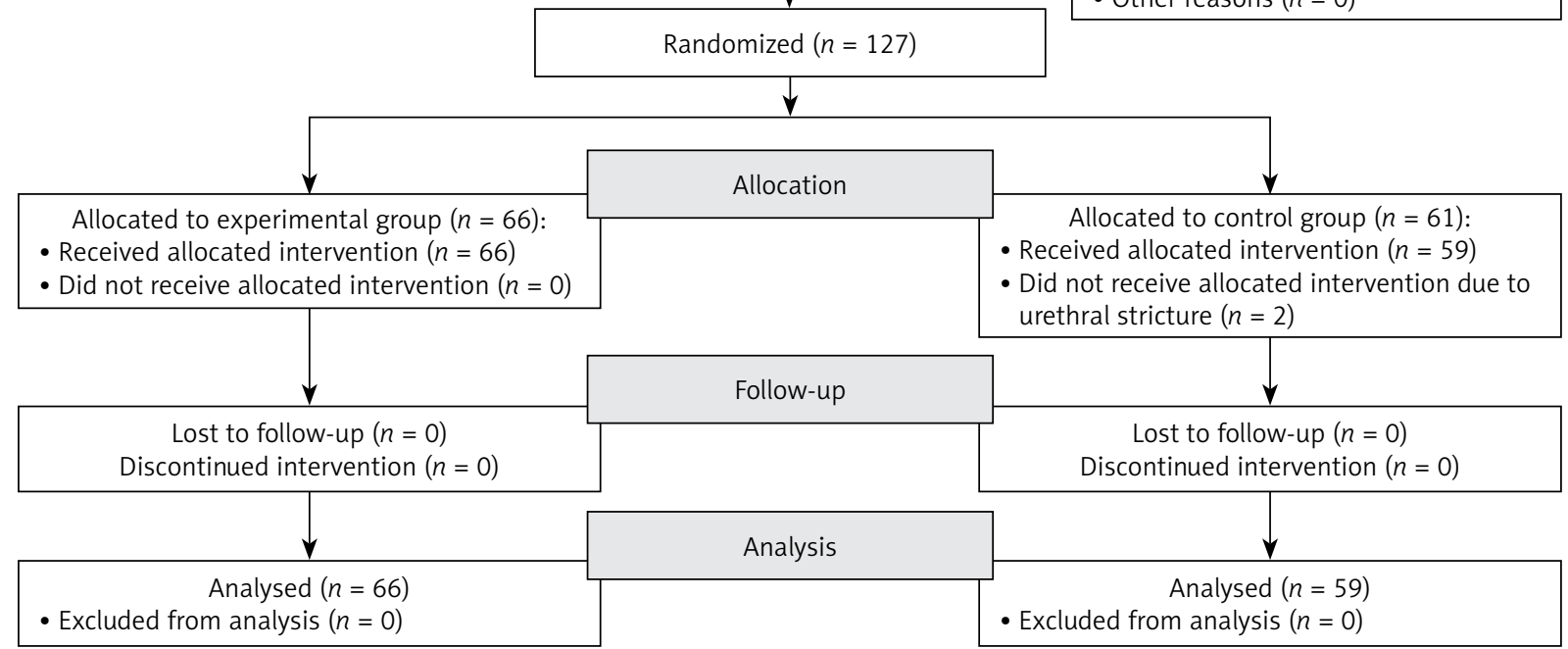

Figure 1. Flow diagram of the progress through the phases of the trial (based on diagram available on http://www.consort-statement.org)

firmed the normal distribution of all variables. The Levene test was applied for assessment of the equality of variances. For the comparison of results between study groups, the $\chi^{2}$ test with the Pearson formula and $t$-test were used for non-parametric and parametric variables, respectively. The differences were considered statistically significant when the $p$-value was $<0.05$.

\section{Results}

The final analysis was based on 125 patients with the mean age of 71.7 years. Two patients from the control group were excluded from the study due to the presence of urethral strictures preventing cystoscopy.

\section{Primary endpoint}

Pain perception assessed directly during the cystoscopy, as well as that assessed after the procedure, did not differ between the two groups. Detailed results are presented in Table II. Each step of the procedure was associated with similar discomfort independently of the anaesthesia of the posterior urethra.

\section{Secondary endpoints}

When evaluated $6 \mathrm{~h}$ after the cystoscopy, patients in the experimental group were less likely to classify pain related the procedure as mild (10.9 vs. $17.5 \%$, relative risk $(R R)=0.60)$ or moderate $(7.3 \%$ vs. $12.3 \%, R R=0.59)$ and more likely to declare

Table II. Pain perception during cystoscopy assessed on 10-point numeric rating scale, mean values and standard deviations

\begin{tabular}{|lcccc|}
\hline Time point/study group & Insertion of catheter & $\begin{array}{c}\text { Insertion } \\
\text { of cystoscope }\end{array}$ & $\begin{array}{c}\text { Second minute } \\
\text { of cystoscopy }\end{array}$ & $\begin{array}{c}\text { Five minutes after } \\
\text { completion } \\
\text { of cystoscopy }\end{array}$ \\
\hline Experimental & $2.75 \pm 2.16$ & $2.93 \pm 2.17$ & $2.38 \pm 1.92$ & $1.59 \pm 1.60$ \\
\hline Control & - & $3.34 \pm 2.22$ & $2.00 \pm 1.65$ & $1.58 \pm 1.25$ \\
\hline Absolute difference & - & 0.41 & -0.38 & -0.01 \\
\hline
\end{tabular}


that the cystoscopy was painless (81.8\% vs. $70.2 \%$, $\mathrm{RR}=1.17)$. The percentage of patients demanding analgesics within $6 \mathrm{~h}$ after the procedure was $0 \%$ in the experimental group and $1.8 \%$ in the control group $(p>0.05)$. During 14 days after the procedure, UTI was diagnosed in $8.1 \%$ of patients in the experimental group and $7.3 \%$ of patients in the control group $(p>0.05)$. The incidence of symptomatic UTIs was $1.6 \%$ vs. $5.4 \%$, respectively $(p>0.05)$. The most common pathogen isolated from urine was Escherichia coli (75\% of symptomatic UTIs), followed by Klebsiella pneumoniae ( $25 \%$ of symptomatic UTIs).

\section{Post-hoc analysis}

Statistically significant differences regarding pain perception were noted depending on the patients' age and number of procedures performed in the past with no relation to the type of anaesthesia. Patients aged $<75$ years declared significantly higher NRS scores at the time of insertion of the cystoscope into the bladder (3.61 vs. 2.62, $p<0.05$ ). With an increasing number of TURBTs performed in the past, NRS scores declared by patients during cystoscopy decreased. The pain perception was significantly lower during catheterisation in patients with a history of at least 2 TURBTs (2.33 vs. $3.44, p<0.05)$, at the second minute of cystoscopy in patients with a history of at least 3 TURBTs (1.92 vs. 2.62, $p<0.05$ ) and during insertion of the cystoscope into the bladder in patients with a history of at least 10 TURBTs (2.21 vs. $3.29, p<0.05)$.

\section{Discussion}

Bladder cancer is the most common malignancy occurring within the urinary tract. According to GLOBOCAN data, the incidence in Central Europe is 15.1 new cases per 100000 habitants per year [1]. About $75 \%$ of patients are diagnosed with non-muscle invasive bladder cancer (NMIBC) [9]. They undergo TURBT followed by intravesical chemo- and/ or immunotherapy. Due to the significant risk of recurrence and progression, these patients need close, often lifelong, follow-up with numerous cystoscopies performed every 3-12 months [2]. Despite significant technological improvement, including wide implementation of flexible cystoscopies, the procedure remains uncomfortable for patients.

We performed a prospective, randomised controlled study aimed at optimisation of anaesthesia in men undergoing cystoscopy. As patients report that the most painful step of the procedure is the moment when the cystoscope passes the posterior urethra $[7,8]$, we focused on improvement in anaesthesia of this particular region. To our knowledge, this is the first study that presents a method of anaesthesia of the posterior urethra and assesses its clinical significance.

Our study did not reveal a direct and statistically significant effect of anaesthesia of the posterior urethra at the time of cystoscopy; however, it did find an important improvement in overall perception of the procedure in the experimental group. This fact can be crucial for patients' compliance. In everyday clinical practice it is not uncommon that patients abandon follow-up because of pain related to cystoscopy. In a historical study by Schrag et al. the incidence of suboptimal frequency of follow-up cystoscopies was over $60 \%$ of NMIBC patients, while almost $10 \%$ of patients did not even have a single follow-up cystoscopy done [10].

Numerous research groups have already attempted to optimise the cystoscopy protocol, especially to reduce pain perception. There have been many prospective, randomized and controlled trials on this issue published in recent years. Thompson et al. and Goel and Aron independently proposed to cool the gel to $4^{\circ} \mathrm{C}[11,12]$; however, the benefit of lower temperature was not confirmed by Bhomi et al. [13]. Brekkan et al. and Holmes et al. independently suggested increasing the volume of lidocaine gel to $20 \mathrm{ml}$ before cystoscopy in men, especially in patients younger than 55 years $[14,15]$. Khan et al. outlined the importance of the delivery rate of lidocaine gel, indicating that slow administration within $10 \mathrm{~s}$ reduces patient discomfort compared to 2-second administration [16]. Vasudeva et al. and Losco et al. independently examined the optimal dwell time of intraurethral lidocaine gel before insertion of the cystoscope. The first group concluded that $15 \mathrm{~min}$ is more effective than both 5 and 10 min for patient as well as physician assessment of pain intensity [17], while the second group did not observe significant differences between immediate insertion of the cystoscope and after a 3-minute delay [18]. Moharari et al. added ketamine to lidocaine gel and observed significantly lower scores on the visual analogue scale compared to lidocaine alone [19]. The analgesic effect can also be strengthened when lidocaine gel is combined with oral 
medication. Komiya et al. proposed administering orally a $160 \mathrm{mg}$ single dose of zaltoprofen $60 \mathrm{~min}$ before cystoscopy, which resulted in lower pain scores compared to no premedication [20]. Gunendran et al. described a "bag squeeze" technique, during which a member of the nursing staff gently squeezes the irrigating fluid bag until the opening of the external sphincter visible on the video camera before the scope passes through the posterior urethra [21]. Zhang et al. recently improved this technique. They confirmed that higher irrigation pressure is associated with less discomfort during cystoscopy and proposed to raise the irrigation solution bag and adjust the height in order to easily improve patients' satisfaction [22]. Apart from technical tips and tricks, Yeo et al. found that listening to classical music during rigid cystoscopy is a simple, economical and effective means of enhancing patient comfort and reducing feelings of pain, anxiety, and dissatisfaction [23].

Finally, numerous research groups have questioned the value of the analgesic effect of lidocaine gel for cystoscopy. In 2008 two meta-analyses on this issue with contradictory results were published. While Aaronson et al. concluded that intraurethral instillation of lidocaine gel provides control of moderate to severe pain and benefit to male patients undergoing cystoscopy [24], Patel et al. provided no evidence of a statistically significant difference in pain scores between lidocaine gel and plain gel instillation [25]. Apart from uncertainty regarding effectiveness, some authors report that delivery of lidocaine gel can cause significant discomfort compared to plain lubricant [26]; however, this finding is not consistent within the literature [15]. None of the already published studies have focused on optimization of anaesthesia of the posterior urethra.

In the present study we found that age and number of cystoscopies performed in the past were inversely correlated with pain intensity during the procedure. This finding was not universal, as statistically significant differences were not observed at all assessed time points, or in all examined groups. However, there is a trend to worse pain scoring during the first cystoscopy and in younger men, which is also described by other authors [3-5, 2022]. While the aim of our study was to optimise local anaesthesia for cystoscopy, these findings are clinically useless despite their relevance. However, being fully consistent with previously published data, they confirm that our methods and protocol are sound, despite the negative findings.

Our study has some limitations. First, nowadays flexible cystoscopy is becoming a standard procedure, while rigid instruments are used less commonly. However, in our institution we still use both rigid and flexible cystoscopes. What is more, we think that in many urological centres rigid cystoscopes are still in use due to their simplicity, reliability and cost-effectiveness. Finally, the character of the instrument does not impact the significance of the results obtained in our study, although one could expect lower NRS scores if a flexible cystoscope were used [27]. Second, as this study is not a blind one, the level of evidence is limited. However, from a practical point of view, it is impossible to plan a blind and especially double-blind study of such character. Last but not least is the fact that as many as six physicians were involved in conducting the experiment. The significance of this fact is substantially reduced by standardisation of the procedure and inclusion of a similar number of patients in both study groups by each resident.

\section{Conclusions}

Anaesthesia of the posterior urethra does not decrease the pain at the time of introducing a cystoscope and has no influence on the overall feeling of pain during the procedure. However, it improves the general perception of the procedure, and hence may positively influence patient compliance. Standard injection of anaesthetic gel through the external urethral orifice to exclude pain perception within the posterior urethra is as sufficient as extended gel distribution to the posterior urethra.

\section{Conflict of interest}

The authors declare no conflict of interest.

\section{References}

1. Torre LA, Bray F, Siegel RL, et al. Global cancer statistics, 2012. CA Cancer J Clin 2015; 65: 87-108.

2. Babjuk M, Böhle A, Burger M, et al. EAU Guidelines on non-muscle-invasive urothelial carcinoma of the bladder: update 2016. Eur Urol 2017; 71: 447-61.

3. Seklehner S, Remzi M, Fajkovic H, et al. Prospective multi-institutional study analyzing pain perception of flexible and rigid cystoscopy in men. Urology 2015; 85: 737-41.

4. Greenstein A, Greenstein I, Senderovich S, Mabjeesh NJ. Is diagnostic cystoscopy painful? Analysis of 1,320 consecutive procedures. Int Braz J Urol 2014; 40: 533-8. 
5. Stein M, Lubetkin D, Taub HC, et al. The effects of intraurethral lidocaine anesthetic and patient anxiety on pain perception during cystoscopy. J Urol 1994; 151: 1518-21.

6. Ellerkmann RM, Dunn JS, McBride AW, et al. A comparison of anticipated pain before and pain rating after the procedure in patients who undergo cystourethroscopy. Am J Obstet Gynecol 2003; 189: 66-9.

7. Taghizadeh AK, El Madani A, Gard PR, et al. When does it hurt? Pain during flexible cystoscopy in men. Urol Int 2006; 76: 301-3.

8. Chen YT, Hsiao PJ, Wong WY, et al. Randomized double-blind comparison of lidocaine gel and plain lubricating gel in relieving pain during flexible cystoscopy. J Endoruol 2005; 19: 163-6.

9. Poletajew S, Biernacki R, Buraczynski P, et al. Stage of bladder cancer in Central Europe - Polish perspective. Neoplasma 2016; 63: 642-7.

10. Schrag D, Hsieh LJ, Rabbani F, et al. Adherence to surveillance among patients with superficial bladder cancer. J Natl Cancer Inst 2003; 95: 588-97.

11. Thompson TJ, Thompson N, O’Brien A, et al. To determine whether the temperature of $2 \%$ lignocaine gel affects the initial discomfort which may be associated with its instillation into the male urethra. BJU Int 1999; 84: 1035-7.

12. Goel R, Aron M. Cooled lignocaine gel: does it reduce urethral discomfort during instillation? Int Urol Nephrol 2003; 35: 375-7.

13. Bhomi KK, Rizal S, Pradhan M, et al. Pain during rigid cystoscopy: a prospective randomized controlled study comparing the benefit of cooled and room temperature lignocaine gel. Nepal Med Coll J 2011; 13: 55-7.

14. Brekkan E, Ehrnebo M, Malmstrom PU, et al. A controlled study of low and high volume anesthetic jelly as a lubricant and pain reliever during cystoscopy. J Urol 1991; 146: 24-7.

15. Holmes M, Stewart J, Rice M. Flexible cystoscopy: is the volume and content of the urethral gel critical? J Endourol 2001; 15: 855-8.

16. Khan MA, Beyzade B, Tau W, et al. Effect of the rate of delivery of lignocaine gel on patient discomfort perception prior to performing flexible cystoscopy. Urol Int 2002; 68: 164-7.

17. Vasudeva P, Kumar A, Kumar N, et al. Effect of intraurethral dwell time of local anesthetic jelly on pain perception in men undergoing outpatient rigid cystoscopy: a randomized prospective study. J Endourol 2014; 28: 846-9.

18. Losco G, Antoniou S, Mark S. Male flexible cystoscopy: does waiting after insertion of topical anaesthetic lubricant improve patient comfort? BJU Int 2011; 108 Suppl. 2: 42-4.

19. Moharari RS, Najafi A, Khajavi MR, et al. Intraurethral instillation of ketamine for male rigid cystoscopy. J Endourol 2010; 24: 2033-6.

20. Komiya A, Endo T, Kobayashi M, et al. Oral analgesia by non-steroidal anti-inflammatory drug zaltoprofen to manage cystoscopy-related pain: a prospective study. Int J Urol 2009; 16: 874-80.

21. Gunendran T, Briggs RH, Wemyss-Holden GD, Neilson D. Does increasing hydrostatic pressure ("bag squeeze") during flexible cystoscopy improve patient comfort: a randomized, controlled study. Urology 2008; 72: 255-8.

22. Zhang ZS, Wang XL, Zeng SX, et al. Pressure makes pleasure: a preliminary study of increasing irrigation pressure of flexi- ble cystoscopy improves male patient comfort by an easy way. J Endourol 2015; 29: 1361-5.

23. Yeo JK, Cho DY, Oh MM, et al. Listening to music during cystoscopy decreases anxiety, pain, and dissatisfaction in patients: a pilot randomized controlled trial. J Endourol 2013; 27: 459-62.

24. Aaronson DS, Walsh TJ, Smith JF, et al. Meta-analysis: does lidocaine gel before flexible cystoscopy provide pain relief? BJU Int 2009; 104: 506-9.

25. Patel AR, Jones JS, Babineau D. Lidocaine $2 \%$ gel versus plain lubricating gel for pain reduction during flexible cystoscopy: a meta-analysis of prospective, randomized, controlled trials. J Urol 2008; 179: 986-90.

26. Ho KJ, Thompson TJ, O’Brien A, et al. Lignocaine gel: does it cause urethral pain rather than prevent it? Eur Urol 2003; 43: 194-6.

27. Krajewski W, Zdrojowy R, Wojciechowska J, et al. Patient comfort during flexible and rigid cystourethroscopy. Videosurgery Miniinv 2016; 11: 94-7.

Received: 22.10.2016, accepted: 23.01.2017. 